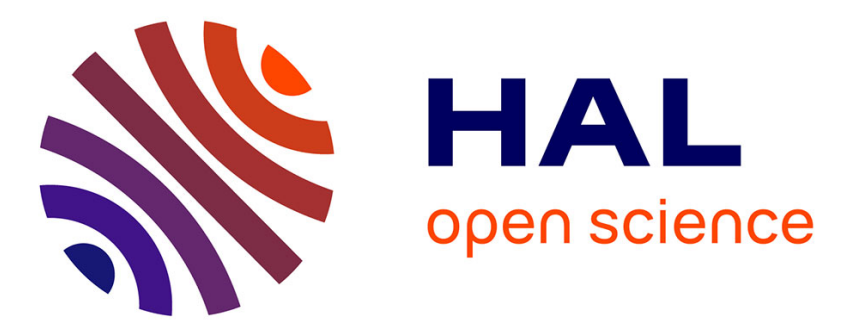

\title{
A low phase noise microwave frequency synthesis for a high-performance cesium vapor cell atomic clock
}

\author{
Bruno Francois, Claudio Calosso, J. M. Danet, Rodolphe Boudot
}

\section{To cite this version:}

Bruno Francois, Claudio Calosso, J. M. Danet, Rodolphe Boudot. A low phase noise microwave frequency synthesis for a high-performance cesium vapor cell atomic clock. Review of Scientific Instruments, 2014, 85 (9), pp.094709. 10.1063/1.4896043 . hal-01232008

\section{HAL Id: hal-01232008 \\ https://hal.science/hal-01232008}

Submitted on 17 Apr 2021

HAL is a multi-disciplinary open access archive for the deposit and dissemination of scientific research documents, whether they are published or not. The documents may come from teaching and research institutions in France or abroad, or from public or private research centers.
L'archive ouverte pluridisciplinaire HAL, est destinée au dépôt et à la diffusion de documents scientifiques de niveau recherche, publiés ou non, émanant des établissements d'enseignement et de recherche français ou étrangers, des laboratoires publics ou privés. 


\title{
A low phase noise microwave frequency synthesis for a high-performance cesium vapor cell atomic clock
}

\author{
B. François, ${ }^{1}$ C. E. Calosso ${ }^{2}$ J. M. Danet ${ }^{3}$ and R. Boudot ${ }^{1}$ \\ ${ }^{1}$ FEMTO-ST, CNRS, Université de Franche-Comté, 26 chemin de l'Epitaphe, 25030 Besançon, France \\ ${ }^{2}$ INRIM, Strada delle Cacce 91, 10135 Torino, Italy \\ ${ }^{3}$ LNE-SYRTE, Observatoire de Paris, CNRS-UPMC, 61 avenue de l'Observatoire, 75014 Paris, France
}

(Received 31 July 2014; accepted 7 September 2014; published online 26 September 2014)

\begin{abstract}
We report the development, absolute phase noise, and residual phase noise characterization of a $9.192 \mathrm{GHz}$ microwave frequency synthesis chain devoted to be used as a local oscillator in a highperformance cesium vapor cell atomic clock based on coherent population trapping (CPT). It is based on frequency multiplication of an ultra-low phase noise $100 \mathrm{MHz}$ oven-controlled quartz crystal oscillator using a nonlinear transmission line-based chain. Absolute phase noise performances of the $9.192 \mathrm{GHz}$ output signal are measured to be $-42,-100,-117 \mathrm{~dB} \mathrm{rad}^{2} / \mathrm{Hz}$ and $-129 \mathrm{~dB} \mathrm{rad}^{2} / \mathrm{Hz}$ at $1 \mathrm{~Hz}, 100 \mathrm{~Hz}, 1 \mathrm{kHz}$, and $10 \mathrm{kHz}$ offset frequencies, respectively. Compared to current results obtained in a state-of-the-art CPT-based frequency standard developed at LNE-SYRTE, this represents an improvement of $8 \mathrm{~dB}$ and $10 \mathrm{~dB}$ at $f=166 \mathrm{~Hz}$ and $f=10 \mathrm{kHz}$, respectively. With such performances, the expected Dick effect contribution to the atomic clock short term frequency stability is reported at a level of $6.2 \times 10^{-14}$ at $1 \mathrm{~s}$ integration time, that is a factor 3 higher than the atomic clock shot noise limit. Main limitations are pointed out. (C) 2014 AIP Publishing LLC. [http://dx.doi.org/10.1063/1.4896043]
\end{abstract}

\section{INTRODUCTION}

Atomic frequency references provide the most stable signals over long integration times because their frequency is determined by an atomic transition. A wide range of industrial and technical fields, such as telecommunication, navigation, defense, and space applications, require stable and reliable timing signals that can be provided only by atomic frequency standards. Vapor cell atomic clocks constitute a valid solution because they combine compactness, reliability, low power consumption, and excellent relative frequency stability. Over the last years, thanks to the progress of semiconductor lasers and the use of dedicated techniques, state-of-theart laboratory-prototype vapor cell atomic clocks, based on optical-microwave double resonance technique ${ }^{1}$ or coherent population trapping (CPT), ${ }^{2}$ have demonstrated short-term frequency stability in the $1-3 \times 10^{-13}$ range, ${ }^{3-7}$ making them competitive for short and mid-term integration times with bulky hydrogen masers. INRIM has developed a pulsed optically pumped $\mathrm{Rb}$ frequency standard with optical detection with a fractional frequency stability of $1.7 \times 10^{-13} \tau^{-1 / 2}$ up to $10000 \mathrm{~s}$ integration time. ${ }^{3}$ LNE-SYRTE has proposed a pulsed Cs vapor cell CPT clock with short term frequency stability of $3.2 \times 10^{-13}$ up to averaging times of $1000 \mathrm{~s}^{4}$ LTF-UNINE has demonstrated a $\mathrm{CW}$ regime $\mathrm{Rb}$ clock with a frequency stability of $1.36-2.4 \times 10^{-13}$ up to averaging times of 1000 s. $^{5-7}$

Up to date, a major contribution to limitation of the short term frequency stability of these high-performance compact vapor cell atomic clocks is the local oscillator (LO) phase noise through the so-called intermodulation effect in continuous $(\mathrm{CW})$ regime clocks ${ }^{8}$ or Dick effect ${ }^{9}, 10$ in pulsed clocks. Indeed, the short-term frequency stability of an atomic clock can be degraded by down conversion of the LO frequency noise at even multiples of the cycle rate into the frequency band of the selective resonator response. In a $\mathrm{CW}$ atomic clock with resonance frequency $v_{0}$, operating at a LO modulation frequency $f_{m}$, the frequency stability limitation $\sigma_{y_{L O}}(\tau)$ due to this aliasing effect is given such as

$$
\sigma_{y_{L O}}^{2}(\tau) \sim \frac{1}{\tau}\left(\frac{f_{m}}{v_{0}}\right)^{2} S_{\varphi}\left(2 f_{m}\right)=\frac{1}{\tau} S_{y}\left(2 f_{m}\right),
$$

where $S_{\varphi}\left(2 f_{m}\right)$ is the power spectral density (PSD) of the local oscillator phase fluctuations in the free-running regime at Fourier frequency $f=2 f_{m} . S_{y}(f)=\frac{f^{2}}{v_{0}^{2}} S_{\varphi}(f)$ is the PSD of the microwave oscillator fractional frequency fluctuations at offset frequency $f$. According to this relation, the development of a Cs atomic clock with a relative frequency stability of $10^{-13}$ at $1 \mathrm{~s}$, operating at a LO modulation frequency $f_{m}=83 \mathrm{~Hz}$, requires a local oscillator with a phase noise at $2 f_{m}=166 \mathrm{~Hz}$ lower than $-99 \mathrm{~dB} \mathrm{rad} / \mathrm{Hz}$. In a pulsed clock, the effect of the LO frequency noise has been characterized by the sensitivity function $g(t)^{10}$ which is the response of the atomic signal to a phase step of the interrogation oscillator at time $t$. The shape of $g(t)$ depends on the atomic system and on the kind of interrogation used. The frequency stability limitation of an atomic clock due to the Dick effect is given from $^{10}$

$$
\sigma_{y_{L O}}^{2}(\tau)=\frac{1}{\tau} \sum_{i=1}^{\infty} \frac{g_{i}^{2}}{g_{0}^{2}} S_{y}\left(i / T_{c}\right)=\frac{1}{\tau} \sum_{i=1}^{\infty} \frac{g_{i}^{2}}{g_{0}^{2}} S_{y}\left(2 i f_{m}\right) .
$$

The parameters $g_{i}$ and $g_{0}$ are defined from the sensitivity function $g .{ }^{10} T_{c}$ is the clock cycle time and $f_{c}=1 / T_{c}$ is the clock cycle frequency. $i / T_{c}$ are the harmonics of the interrogation frequency. For the pulsed Cs CPT clock developed in LNE-SYRTE, the clock cycle time is $T_{c}=6 \mathrm{~ms}$, i.e., 
$f_{c}=166 \mathrm{~Hz}$. Additionally, compared to classical clocks based on a two-level atomic system, it has been recently demonstrated that the pulsed CPT clock Allan deviation exhibits a higher sensitivity to high frequency components of the LO noise. $^{4}$

Reducing the Dick effect contribution requires the development of ultra-stable and low phase noise local oscillators. The purest microwave signals are nowadays obtained through optical-microwave frequency division with optical frequency combs $^{11,12}$ or the use of cryogenic sapphire oscillators ${ }^{13}$ and associated frequency synthesis. ${ }^{14}$ Nevertheless, these systems remain voluminous, complex, and are not compatible with compactness requirements of vapor cell atomic clocks. In this domain, the generation of microwave signals referenced to an ultra-low noise quartz crystal oscillator remains a welladapted solution. ${ }^{15-17}$

In the frame of the EURAMET MClocks project, ${ }^{18}$ this paper describes the development of a microwave $9.192 \mathrm{GHz}$ frequency synthesis chain for a high-performance CPT clock. It is inspired from Ref. 16 but different component references are used for some key steps. Absolute phase noise and predicted Dick effect contributions are reported whereas only residual phase noise was given in Ref. 16. Compared to Ref. 16, the synthesis residual noise is slightly degraded in the $100-1 \mathrm{kHz}$ range. This is attributed to the use of newgeneration nonlinear transmission line (NLTL) comb generators that exhibit excess flicker noise. At the opposite, the present synthesis residual noise floor is about $10 \mathrm{~dB}$ better mainly thanks to a new-design phase lock loop (PLL) electronics (see Sec. II). Additionally, a $4.596 \mathrm{GHz}$ output signal is generated from the $9.192 \mathrm{GHz}$ signal to be used in a simple-architecture pulsed Cs CPT clock in which a pigtailed electro-optic modulator is used to detect Ramsey-CPT fringes. ${ }^{19}$ Most significantly, thanks to the use of an ultra-low phase oven-controlled quartz crystal oscillator (OCXO) pilot, a relevant 4-13 dB improvement of absolute phase noise performances in the $100 \mathrm{~Hz}-1 \mathrm{kHz}$ offset frequency range is obtained compared to the current system used in LNE-SYRTE. The Dick effect contribution limitation to the clock frequency instability is reduced at a level of $6.2 \times 10^{-14}$.

\section{ARCHITECTURE OF THE FREQUENCY SYNTHESIS CHAIN}

Figure 1 shows a detailed scheme of the microwave frequency synthesizer architecture. The pilot and key element of the synthesis chain is a $100 \mathrm{MHz}$ oven-controlled quartz crystal oscillator (Pascall OCXOF-E-100) ${ }^{20}$ This source exhibits ultra-low phase noise in the $1 \mathrm{~Hz}-1 \mathrm{MHz}$ Fourier frequency range and is of relevant interest for compact vapor cell atomic clocks applications. The output power of the OCXO is $+19 \mathrm{dBm}$. Its tuning voltage-frequency sensitivity was measured to be $500 \mathrm{~Hz} / \mathrm{V}$. Its tuning voltage-output power dependence was measured to be cancelled at the first order around a tuning voltage of $5 \mathrm{~V}$. The output $100 \mathrm{MHz}$ signal is split into two arms. In the first arm, reference signals at $100 \mathrm{MHz}$ and $10 \mathrm{MHz}$ using a digital frequency divider (Zarlink SP8401) are generated. In the second and main arm, the output $100 \mathrm{MHz}$ signal is frequency-doubled to $200 \mathrm{MHz}$

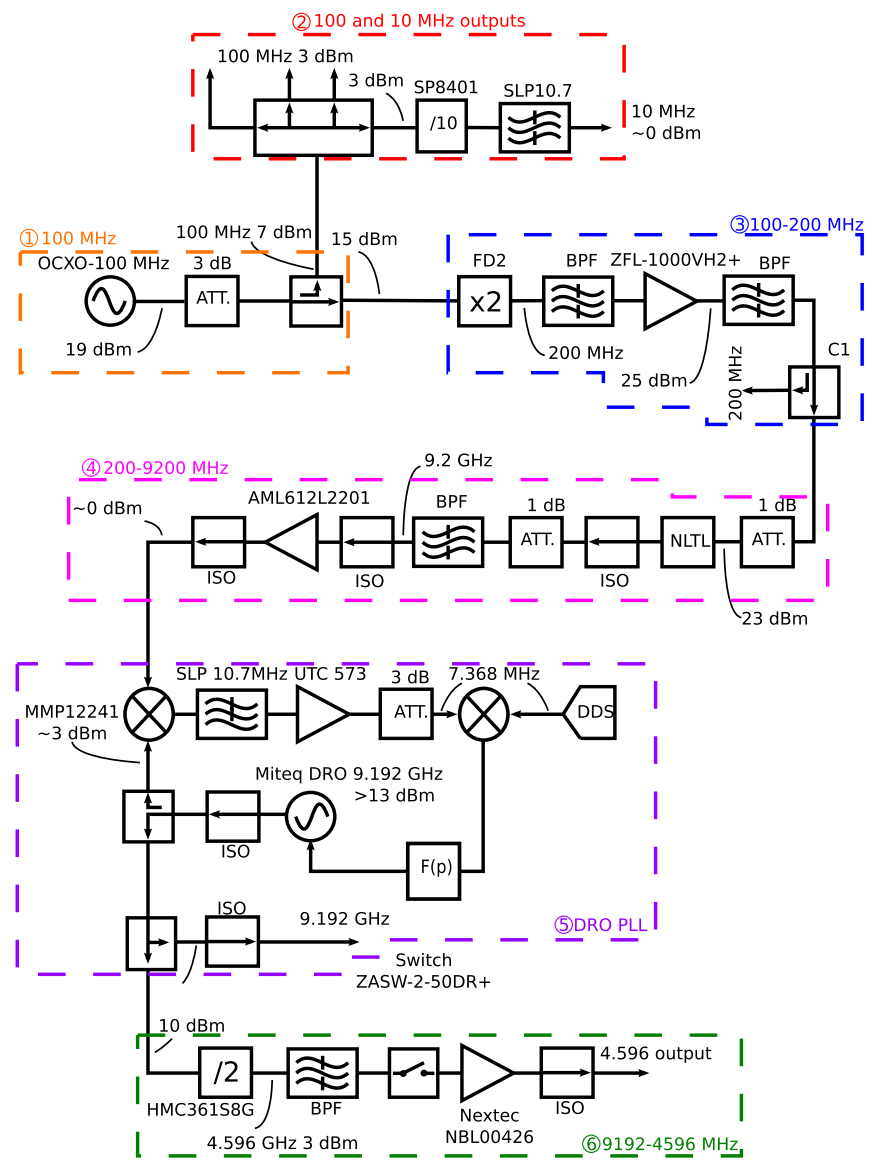

FIG. 1. Architecture of the frequency synthesis chain. 6 main blocks are indicated on the diagram for better understanding. 1: the $100 \mathrm{MHz}$ OCXO pilot, 2: $100 \mathrm{MHz}$ and $10 \mathrm{MHz}$ outputs, 3: 100-200 MHz frequency doubling stage, 4: NLTL-based 200-9200 MHz chain, 5: $9.192 \mathrm{GHz}$ DRO phase lock loop, 6: 9.192-4.596 GHz frequency division chain.

using a passive frequency-doubler (Mini-Circuits FD2). The output $200 \mathrm{MHz}$ signal is bandpass filtered and amplified to a power of $23 \mathrm{dBm}$ with a low noise amplifier (Minicircuits ZFL-1000VH2+). The $200 \mathrm{MHz}$ signal is used to drive a NLTL comb generator device (Picosecond Model 7110) that generates harmonics up to about $20 \mathrm{GHz}$. The $9.2 \mathrm{GHz}$ harmonic output is bandpass-filtered with a $50 \mathrm{MHz}$-bandwidth bandpass filter and amplified to a power of about $0 \mathrm{dBm}$ with a low noise microwave amplifier (AML612L2201). Microwave isolators and attenuators are added at the output of the NLTL to help for impedance matching, optimization of the phase noise performances, and to prevent undesired feedback. The NLTL component was found to be a critical element. Setting the optimal configuration that optimizes phase noise performances of this synthesis chain section can require patience and numerous experimental tests. Microwave isolators were selected for their high isolation properties. Optimization of impedance matching of NLTL input and output was found to be important. Once the best configuration is found, we observed excellent repeatability and stability of performances along time. The output $9.2 \mathrm{GHz}$ is mixed with the $9.192 \mathrm{GHz}$ signal coming from a dielectric resonator oscillator (DRO MITEQ-DRO-G-09192-MT \pm 140 ), to produce a frequency beatnote at $7.368 \mathrm{MHz}$. The latter is low-pass 


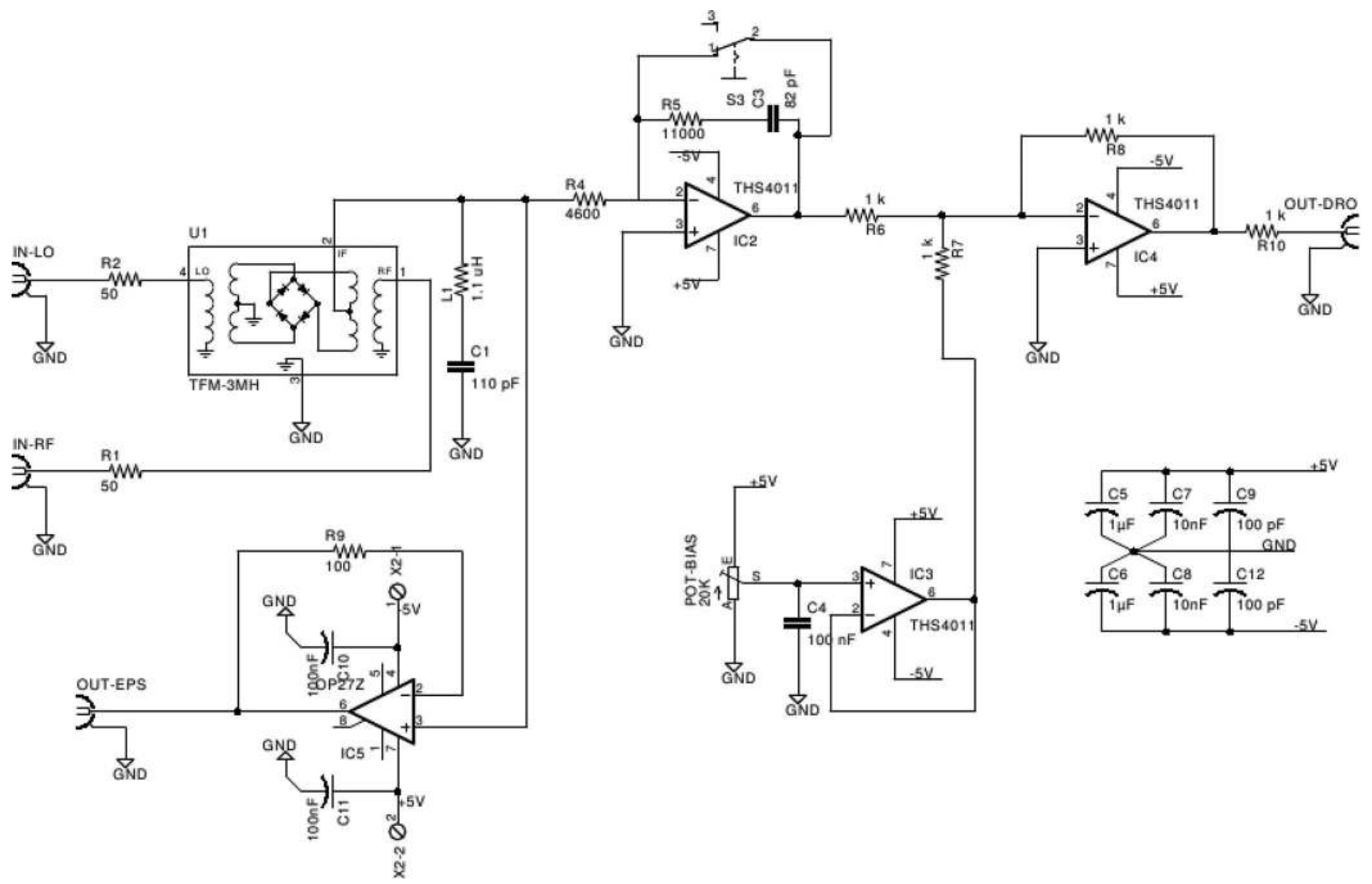

FIG. 2. Schematic of the DRO phase lock servo loop.

filtered, amplified with a high-isolation radiofrequency amplifier (Avantek UTC-573), and compared to the $7.368 \mathrm{MHz}$ signal from a direct digital synthesis (DDS Agilent 33220A). The DDS is referenced by a $10 \mathrm{MHz}$ signal generated from the initial $100 \mathrm{MHz}$ OCXO. The DRO is phase-locked to the frequency-multiplied $100 \mathrm{MHz}$ signal with a bandwidth of about $1 \mathrm{MHz}$ using the circuit described in Figure 2. Both input signals are mixed with a double balanced mixer (MiniCircuits TFM-3MH) with a sensitivity of $0.225 \mathrm{~V} / \mathrm{rad}$. The resulting error signal is filtered, processed in a proportionalintegral (PI) controller, summed with an offset voltage to fix the OCXO bias point, and eventually used to phase-lock the DRO. High speed operational amplifiers (THS4011) are used to ensure proper and high-bandwidth lock of the DRO. An additional OP27 amplifier is used to monitor the error signal. A low phase noise $9.192 \mathrm{GHz}$ signal with a microwave power of about $13 \mathrm{dBm}$ is then obtained at the output of the DRO. This signal is split into two different arms. In the first arm, the $9.192 \mathrm{GHz}$ arm is ready to use. In the other arm, the $9.192 \mathrm{GHz}$ signal is frequency-divided by 2 with a low noise microwave frequency divider (Hittite HMC36158G). The $4.596 \mathrm{GHz}$ output microwave signal is bandpass filtered and amplified with a 4-12 GHz bandwidth high-power amplifier (Nextec NBL-00426) to produce a microwave power of about 20-22 dBm. A 5-GHz bandwidth switch (Minicircuits ZASW-50-DR2+) is implemented in this arm to make possible fast and high-extinction switching of the $4.596 \mathrm{GHz}$ microwave signal. ${ }^{19}$

\section{ABSOLUTE PHASE NOISE MEASUREMENTS}

Measurements are performed with a signal source analyzer (Agilent E5052B) using cross-correlation techniques for enhanced sensitivity. ${ }^{21-23}$ The phase noise uncertainty at effective offset frequencies from $1 \mathrm{~Hz}$ to $100 \mathrm{MHz}$ is given to be about $\pm 5 \mathrm{~dB} .^{23,24}$ This uncertainty has to be considered for phase noise plots of Figures 3, 4, and 8. Figure 3 plots

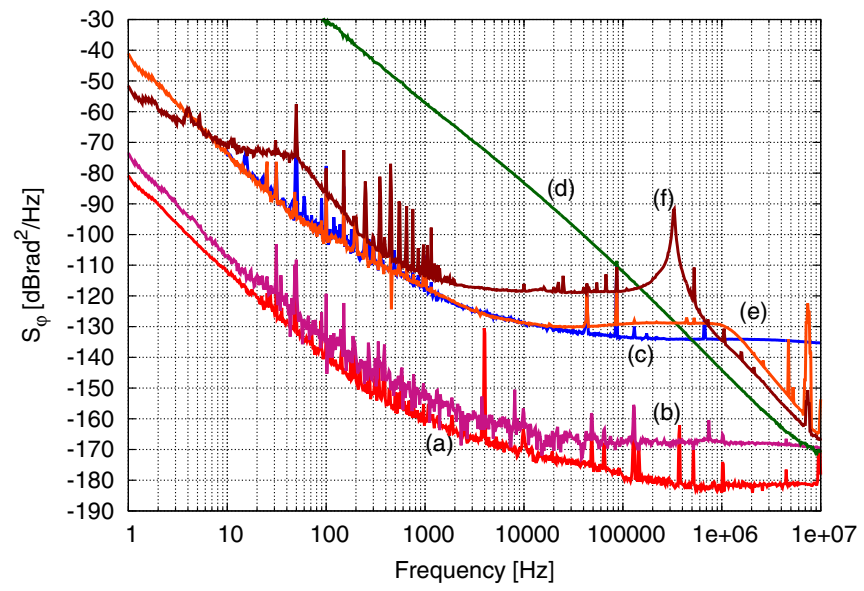

FIG. 3. Absolute phase noise performances of key signals of the synthesis chain. (a) $100 \mathrm{MHz}$ OCXO, (b) $200 \mathrm{MHz}$ signal at the output of the ZFL$1000 \mathrm{VH} 2+$ amplifier (not connected to NLTL-chain), (c) $9200 \mathrm{MHz}$ signal at the output of the NLTL-based chain, (d) free-running DRO at $9.192 \mathrm{GHz}$, (e) locked DRO at $9.192 \mathrm{GHz}$, (f) synthesis output at $9.392 \mathrm{GHz}$ in LNE-SYRTE. 


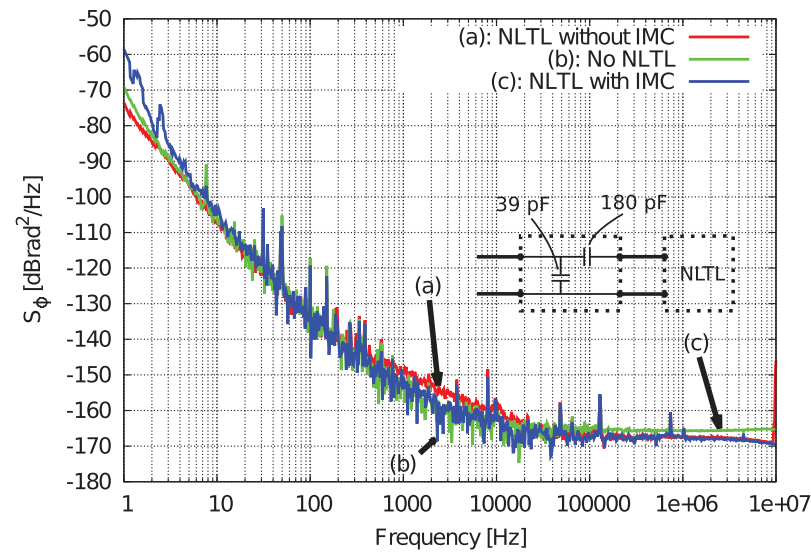

FIG. 4. Absolute phase noise of the $200 \mathrm{MHz}$ signal in three configurations. (a) NLTL connected to the $200 \mathrm{MHz}$ stage output without IMC. (b) NLTLchain disconnected from the $200 \mathrm{MHz}$ chain. (c) NLTL connected to the $200 \mathrm{MHz}$ stage output with IMC.

absolute phase noise performances of key signals of the synthesis chain at 100, 200, 9200, and $9192 \mathrm{MHz}$ (free-running or locked DRO). The phase noise of the $9.192 \mathrm{GHz}$ signal used in LNE-SYRTE CPT clock is shown for comparison. The phase noise spectrum of the free-running $100 \mathrm{MHz}$ Pascall OCXO is given in $\mathrm{dB} \mathrm{rad} / \mathrm{Hz}$ by the power law $S_{\varphi}(f)$ $=\sum_{i=0}^{-4} b_{i} f^{i}$ with $b_{0}=-182, b_{-1}=-130$, and $b_{-3}=-82$. These performances are excellent and are found to be in the $f=1 \mathrm{~Hz}-10 \mathrm{kHz}$ frequency range significantly better than other OCXOs we tested. For $f<10 \mathrm{kHz}$, the phase noise of the $200 \mathrm{MHz}$ output signal is measured to be as expected $6 \mathrm{~dB}$ higher than the $100 \mathrm{MHz}$ signal phase noise. For $f>10 \mathrm{kHz}$, the phase noise of the output $200 \mathrm{MHz}$ signal is degraded compared to the ideally multiplied $100 \mathrm{MHz}$ signal. The white phase noise floor of the $200 \mathrm{MHz}$ signal is measured to be $-168 \mathrm{~dB} \mathrm{rad}^{2} / \mathrm{Hz}$ and is limited by the input power of the ZFL-1000VH2+ amplifier. ${ }^{25}$ The $9200 \mathrm{MHz}$ signal phase noise is $-42,-100,-130$, and $-135 \mathrm{~dB} \mathrm{rad}^{2} / \mathrm{Hz}$ at $1 \mathrm{~Hz}, 100 \mathrm{~Hz}, 10 \mathrm{kHz}$, and $1 \mathrm{MHz}$ offset, respectively. Except for the $200 \mathrm{~Hz}-1 \mathrm{kHz}$ region where an excess noise degradation of about $3 \mathrm{~dB}$ attributed to the NLTL residual phase noise is observed, the signal frequency multiplication from $200 \mathrm{MHz}$ to $9200 \mathrm{MHz}$ causes a 33-dB increase of the noise as expected.

Interestingly, we observed that impedance mismatching between the $200 \mathrm{MHz}$ stage output and the $200-9200 \mathrm{MHz}$ NLTL-based chain can perturbate significantly the absolute phase noise of the $200 \mathrm{MHz}$ signal. To highlight this point, Figure 4 shows the absolute phase noise of the $200 \mathrm{MHz}$ signal in different situations. For curve (a), the $200 \mathrm{MHz}$ stage output is connected to the NLTL-based chain while the $200 \mathrm{MHz}$ signal is measured through the coupler C1 (see Figure 1). For curve (b), the signal is directly measured at the $50 \Omega$ impedance-matched output of the $200 \mathrm{MHz}$ bandpass filter while the NLTL-chain section is removed. In the latter case, we observe a relevant improvement (up to $4 \mathrm{~dB}$ ) of the absolute phase noise at $200 \mathrm{MHz}$ in the $200 \mathrm{~Hz}-20 \mathrm{kHz}$ offset range. Simultaneously, the white noise floor is slightly degraded by about $3 \mathrm{~dB}$ in the last case.
For further investigation, we measured the input impedance at $200 \mathrm{MHz}$ of the NLTL component using a network analyzer. The complex impedance of the NLTL with associated connectors was measured to be $z_{\text {NLTL }}=7+22 j$, that is far to be compatible with required $50 \Omega$ impedance matching. In this case, the magnitude of the reflection coefficient $S_{11}$ is only $-2 \mathrm{~dB}$, meaning that $79 \%$ of the RF power of the $200 \mathrm{MHz}$ output stage is reflected back by the NLTL. For improvement, a basic impedance matching L-network circuit (IMC) with lumped elements, shown as an inset in Figure 4, was designed $^{26}$ in order to match the NLTL input impedance to the $200 \mathrm{MHz}$ stage output. With this circuit, the magnitude of the reflection coefficient $S_{11}$ was improved to be $-15 \mathrm{~dB}$. As shown on Figure 4 curve (c), in presence of the NLTL chain, the absolute phase noise of the $200 \mathrm{MHz}$ signal was then improved in the $200 \mathrm{~Hz}-20 \mathrm{kHz}$ range while achieving optimal phase noise floor value.

In the free-running regime, the DRO exhibits a phase noise of $-112 \mathrm{~dB} \mathrm{rad} / \mathrm{Hz}$ at $f=100 \mathrm{kHz}$. Its phase noise floor for $f>10 \mathrm{MHz}$ is $-172 \mathrm{~dB} \mathrm{rad}^{2} / \mathrm{Hz}$ (not shown here). In the locked regime, the $9.192 \mathrm{GHz}$ output signal exhibits phase noise performances of $-42,-100,-129$, and $-130 \mathrm{~dB} \mathrm{rad}^{2} / \mathrm{Hz}$ at $f=1 \mathrm{~Hz}, 100 \mathrm{~Hz}, 10 \mathrm{kHz}$, and $1 \mathrm{MHz}$, respectively. The peak at $7.3 \mathrm{MHz}$ is the signal frequency generated by the DDS included in the DRO PLL. A phase noise reduction of $4-13 \mathrm{~dB}$ is measured in the $100 \mathrm{~Hz}-1 \mathrm{kHz}$ range compared to the current synthesis used in LNE-SYRTE, Paris $^{4}$ (see Fig. 3, curve (f)). Moreover, the number of spurious is greatly reduced in the present synthesis. While not reported for clarity of the figure, phase noise performances of the $4.596 \mathrm{GHz}$ signal were measured to be in good agreement with performances obtained at $9.192 \mathrm{GHz}(6 \mathrm{~dB}$ reduction of the noise). The phase noise floor of the $4.596 \mathrm{GHz}$ signal is limited for $f>1 \mathrm{MHz}$ by the frequency divider residual noise at a level of $-146 \mathrm{~dB} \mathrm{rad} / \mathrm{Hz}$.

\section{RESIDUAL PHASE NOISE MEASUREMENTS AND LIMITATIONS}

Residual phase noise measurements of key components of the frequency synthesis chain were realized. For this purpose, a standard Schottky-diode saturated double-balanced mixer working as a phase detector is used in association with a FFT analyzer (HP 3562A) in single-channel configuration. The uncertainty associated with the measurement of phase noise is estimated to be $\pm 2 \mathrm{~dB}$. Figure 5 shows the residual phase noise of main components in the frequency synthesis chain. All measurements are reported to a carrier frequency of $9.2 \mathrm{GHz}$ and compared to the ideally frequencymultiplied OCXO signal. We observe that the residual noise of the microwave mixer and the microwave amplifier are well below the phase noise of the $100 \mathrm{MHz}$ OCXO. The residual phase noise of the frequency doubler (FD2) is low enough not to degrade the initial OCXO $100 \mathrm{MHz}$ signal for $f<10 \mathrm{kHz}$. On this figure, the residual noise floor of the doubler reported at $9.2 \mathrm{GHz}$ is $-130 \mathrm{~dB} \mathrm{rad}^{2} / \mathrm{Hz}$, that becomes $-163.2 \mathrm{~dB} \mathrm{rad}^{2} / \mathrm{Hz}$ when scaled down at $200 \mathrm{MHz}$. The doubler noise floor was found to be in fact limited by the noise measurement setup at $200 \mathrm{MHz}$. Observing that the absolute 


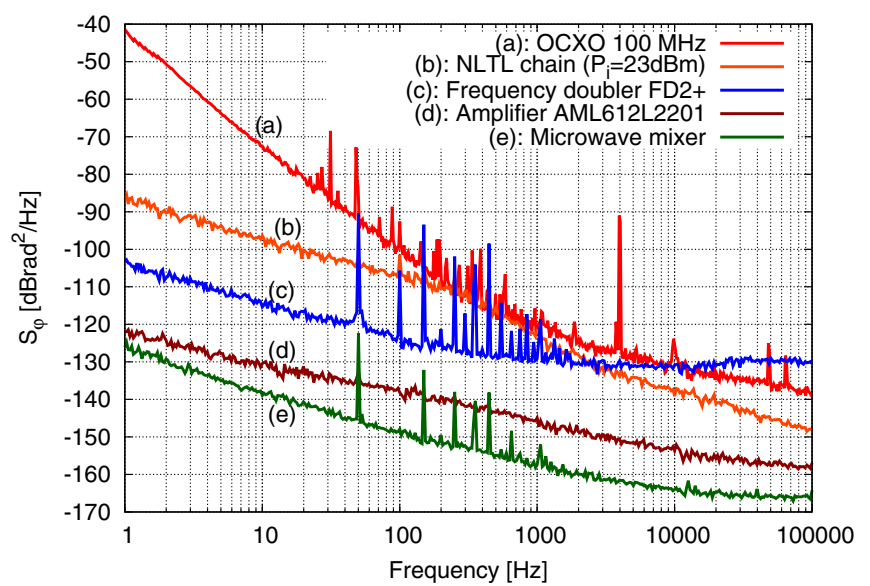

FIG. 5. Residual phase noise reported at $9200 \mathrm{MHz}$ of key components of the synthesis chain. These measurements are compared to the ideal absolute phase noise of the $100 \mathrm{MHz}$ OCXO reported at $9200 \mathrm{MHz}$ to highlight main limitations of the chain. (a) OCXO $100 \mathrm{MHz}$, (b) NLTL chain (input power $P_{i}=23 \mathrm{dBm}$ ), (c) Frequency doubler FD2+ (output referred), (d) Microwave amplifier, (e) Microwave mixer.

phase noise floor at $200 \mathrm{MHz}$ is $-168 \mathrm{~dB} \mathrm{rad}^{2} / \mathrm{Hz}$ (see Fig. 3), we can assume that the actual residual noise floor of the frequency doubler is at least $5 \mathrm{~dB}$ better than the value reported on Fig. 5. To conclude on the limitations of the synthesis, we find that the $9.192 \mathrm{GHz}$ output signal white noise floor is degraded for $f>10 \mathrm{kHz}$ by about $6 \mathrm{~dB}$ by the frequency doubling stage residual noise floor. In the $100 \mathrm{~Hz}-1 \mathrm{kHz}$ offset frequency range, it is measured that the NLTL chain residual phase noise remains a limitation to the ideal frequency multiplication of the OCXO $100 \mathrm{MHz}$ signal.

Further words and independent analysis on the NLTL component are now reported. Figure 6 reports the residual phase noise of the NLTL-based $200-9200 \mathrm{MHz}$ frequency multiplication chain for different input power of the NLTL component. In this experiment, the NLTL-based chain residual phase noise is decreased with decreased input power. The best measurement is obtained for an input power of $23 \mathrm{dBm}$. In this case, the phase noise spectrum exhibits different slopes, $f^{-1}$ for $1 \mathrm{~Hz}<f<100 \mathrm{~Hz}, f^{-2}$ for $100 \mathrm{~Hz}$

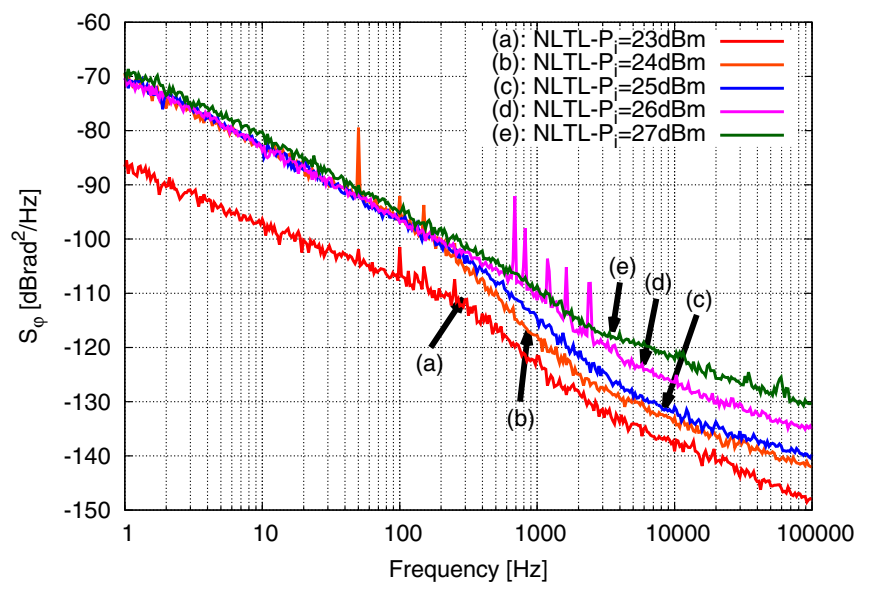

FIG. 6. Residual phase noise at $9200 \mathrm{MHz}$ of the NLTL-based frequency multiplication chain versus the NLTL input power. $<f<10 \mathrm{kHz}$, and $f^{-1}$ again far from the carrier. This behavior is not clearly explained and was observed on 4 different tested NLTLs. With increased NLTL input power, we observe up to $15 \mathrm{~dB}$ of degradation of the chain residual phase noise in the $100 \mathrm{~Hz}-1 \mathrm{kHz}$ offset range. The NLTL input power for best phase noise performances needs to be found experimentally.

For information, compared to old-generation NLTLs, ${ }^{16}$ we note that the new-generation NLTL presents several relevant drawbacks. It is more expensive, needs to be driven with higher RF power (23-27 dBm instead of 19-23 dBm) and exhibits a residual phase noise about $10 \mathrm{~dB}$ higher. Note that in Ref. 27, the residual phase noise of Step Recovery Diodes (SRD) was measured to be $-120 \mathrm{~dB} \mathrm{rad} / \mathrm{Hz}$ at $f=100 \mathrm{~Hz}$ for a carrier of $10 \mathrm{GHz}$, that is about $10 \mathrm{~dB}$ better than the residual phase noise of our NLTL comb generator. These components could be good candidates for improved performances of the synthesis chain, mainly in the $100-1 \mathrm{kHz}$ offset frequency range. Another solution could be to reduce largely the excess flicker noise of the NLTL using the phase noise suppression technique proposed in Ref. 27.

\section{DICK EFFECT CONTRIBUTION}

In atomic clocks based on CPT physics, the microwave interrogation signal of the LO is optically carried. Consequently, it has to be checked that there is no phase noise degradation between the microwave synthesis output and the optical beatnote that actually interrogates the atoms. Different techniques can be proposed to generate optical sidebands required for CPT interaction. In LNE-SYRTE, both optical lines frequency-splitted by $9.192 \mathrm{GHz}$ are generated using two phase-locked lasers. ${ }^{16}$ In FEMTO-ST, as resumed by Figure 7, CPT optical sidebands are generated by driving a pigtailed $895 \mathrm{~nm}$ Mach-Zehnder intensity EOM at $4.596 \mathrm{GHz}{ }^{19}$ In this setup, an original microwave synchronous detector (not shown here) is implemented to stabilize the optical carrier suppression at the output of the EOM. ${ }^{28}$

Figure 8 reports absolute phase noise performances at $9.192 \mathrm{GHz}$ (a) and corresponding Dick effect contribution on the atomic clock short term frequency stability (b) for

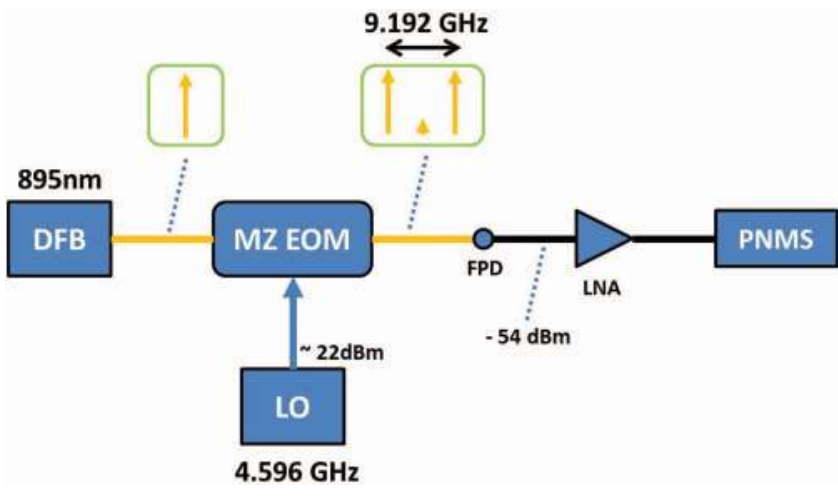

FIG. 7. Generation of the $9.192 \mathrm{GHz}$ optically carried microwave signal by modulating at $4.596 \mathrm{GHz}$ a DFB laser with a pigtailed Mach-Zehnder electrooptic modulator (MZ EOM). The dc bias voltage of the EOM (not shown here) is actively adjusted to stabilize the optical carrier suppression at the output of the EOM. ${ }^{28}$ FPD: fast photodiode, LNA: low noise amplifier. 


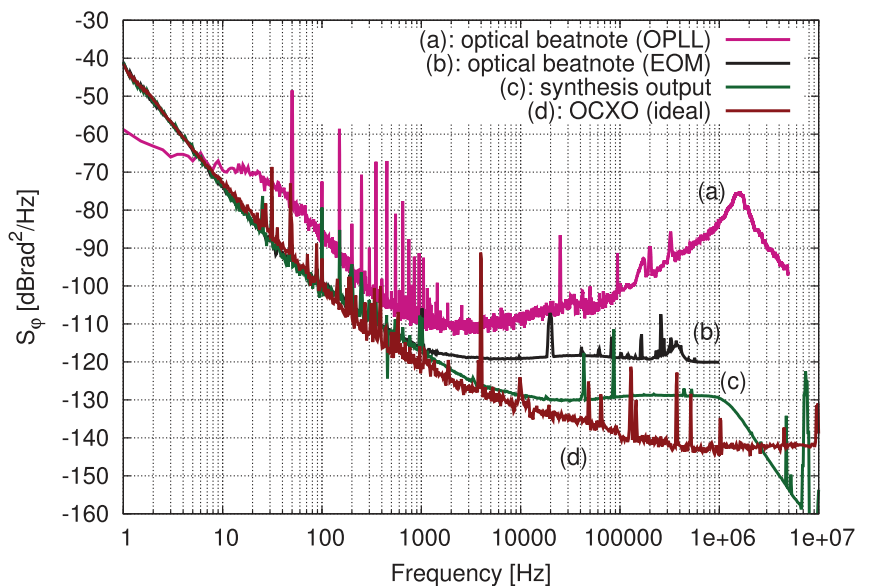

(a)

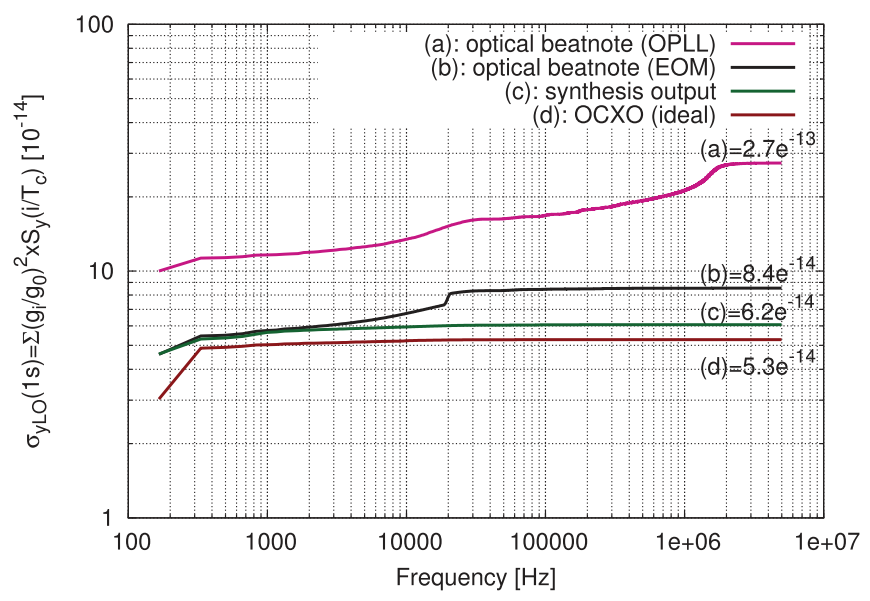

(b)

FIG. 8. Absolute phase noise at $9.192 \mathrm{GHz}$ (a) and expected Dick effect contribution to the CPT atomic clock short term frequency stability (b). Curves are: (a) Optical beatnote signal using 2 phase locked lasers (LNE-SYRTE setup), (b) optical beatnote using an EOM (noise floor limitation due to the measurement setup), (c) output from the present synthesis chain, (d) OCXO ideally frequency-multiplied.

different cases noted (a)-(d). The latter contribution is calculated using Eq. (2). Operating parameters and conditions of the pulsed sequence are reported in Ref. 4. The Dick effect contribution is plotted as a function of the noise integration bandwidth. Once this integrated noise stops to contribute to the Dick effect, $\sigma_{y}$ reaches a floor that is the total Dick effect contribution.

Case (a) is the phase noise of the $9.192 \mathrm{GHz}$ optical beatnote generated from the optical phase lock loop (OPLL) used in LNE-SYRTE. In this system, the $2 \mathrm{MHz}$-bandwidth OPLL degrades greatly the interrogation signal phase noise floor seen by the atoms. The Dick effect contribution is reaching $2.7 \times 10^{-13}$ in a $2 \mathrm{MHz}$ bandwidth. ${ }^{4}$ Case (b) is the phase noise of the $9.192 \mathrm{GHz}$ optical beatnote at the output of the EOM using the setup described in Fig. 7 while case (c) is the phase noise of the $9.192 \mathrm{GHz}$ signal at the direct synthesis output. In this system, no phase noise degradation is observed between the $9.192 \mathrm{GHz}$ optical beatnote and the direct synthesis output for $f<1 \mathrm{kHz}$. For $f>1 \mathrm{kHz}$, the phase noise floor of the $9.192 \mathrm{GHz}$ optical beatnote is limited at a level of
$-118 \mathrm{~dB} \mathrm{rad}^{2} / \mathrm{Hz}$. We checked that this limit is intrinsically due to the phase noise measurement setup (PNMS), more precisely by the low input power $(\sim-54 \mathrm{dBm})$ of the microwave amplifier $^{25}$ placed at the output of the fast photodiode used to detect the optical beatnote. In the EOM-based CPT clock, there is no noise to be integrated for frequencies higher than $20 \mathrm{kHz}$. In case (b), the Dick effect contribution is reported at $8.4 \times 10^{-14}$. Neglecting the limitation due to the PNMS (case (c)), the latter is further reduced at the level of $6.2 \times 10^{-14}$. This value is a factor 3 higher than the atomic clock shot noise limit. It has been calculated that the $3 \mathrm{~dB}$-degradation due to the DRO PLL has no impact on the Dick effect limitation.

In order to evaluate ultimate achievable performances of this chain, case (d) reports the phase noise spectrum of the OCXO $100 \mathrm{MHz}$ signal ideally multiplied to $9.192 \mathrm{GHz}$. In this ideal case, the clock frequency stability limitation would be slightly further reduced at $5.3 \times 10^{-14}$. Note that removing totally spurious lines from the OCXO phase noise spectrum, the ultimate Dick effect contribution of the OCXO would be minimized to a level of $3.3 \times 10^{-14}$, a value close to the atomic clock shot noise limit.

\section{CONCLUSIONS}

We reported the development of a high-performance microwave frequency synthesis chain driven by an ultra-low phase noise $100 \mathrm{MHz}$ OCXO. Key steps are a 100-200 MHz frequency multiplication stage, a 200-9200 MHz NLTL-based chain, and the phase lock of a DRO onto the frequencymultiplied $100 \mathrm{MHz}$ signal. Absolute phase noise performances at $9.192 \mathrm{GHz}$ are found to be $-100 \mathrm{~dB} \mathrm{rad}^{2} / \mathrm{Hz}$ and $-119 \mathrm{~dB} \mathrm{rad}^{2} / \mathrm{Hz}$ at $f=100 \mathrm{~Hz}$ and $f=1 \mathrm{kHz}$, respectively. Performances of the synthesis chain are mainly limited by the NLTL-based chain residual noise in the $100 \mathrm{~Hz}-1 \mathrm{kHz}$ offset frequency range ( $3 \mathrm{~dB}$ degradation). The white noise floor is limited by the $100-200 \mathrm{MHz}$ frequency doubling stage residual noise (6 $\mathrm{dB}$ degradation) and the DRO phase lock loop (3 $\mathrm{dB}$ degradation). Impedance matching issues between the $200 \mathrm{MHz}$ output stage and the $200-9200 \mathrm{MHz}$ NLTL-chain section were pointed out, impacting on the synthesis phase noise performances. No absolute phase noise degradation was found between the direct synthesis output and the optical beatnote at the output of a MZ EOM for $f<1 \mathrm{kHz}$. In present configuration, the Dick effect contribution is reported at the level of $6.2 \times 10^{-14}$. This could be further lowered at 5.3 $\times 10^{-14}$ if the OCXO spectral purity is perfectly transferred to $9.192 \mathrm{GHz}$ and even further reduced at $3.3 \times 10^{-14}$ if the final phase noise spectrum presents no spurious lines. Performances demonstrated are fully compatible with the development of a high-performance $\mathrm{Cs}$ vapor cell atomic clock with expected relative frequency stability better than $10^{-13}$ at $1 \mathrm{~s}$ integration time.

\section{ACKNOWLEDGMENTS}

This work has been funded by the EMRP program (IND55 Mclocks). The EMRP is jointly funded by the EMRP participating countries within EURAMET and the European 
Union (EU). This work was partly supported by LNE, LabeX FIRST-TF, and EquipX Osc-Imp. The authors thank Y. Gruson and E. Rubiola for fruitful discussions.

${ }^{1}$ J. Vanier and C. Mandache, Appl. Phys. B 87, 565 (2007).

${ }^{2}$ J. Vanier, Appl. Phys. B 81, 421 (2005).

${ }^{3}$ S. Micalizio, C. E. Calosso, A. Godone, and F. Levi, Metrologia 49, 425 (2012).

${ }^{4}$ J. M. Danet, M. Lours, S. Guérandel, and E. De Clercq, IEEE Trans. Ultrason. Ferroelectr. Freq. Control 61(4), 567 (2014).

${ }^{5}$ T. Bandhi, C. Affolderbach, C. Calosso, and G. Mileti, Electron. Lett. 47(12), 698 (2011).

${ }^{6}$ C. Stefanucci, T. Bandi, F. Merli, M. Pellaton, C. Affolderbach, G. Mileti, and A. K. Skrivervik, Rev. Sci. Instrum. 83, 104706 (2012).

${ }^{7} \mathrm{~T}$. Bandhi et al., in Proceedings of the International Frequency Control Symposium, Baltimore, 2012 (IEEE, 2012), pp. 1-6.

${ }^{8}$ C. Audoin, V. Candelier, and N. Dimarcq, IEEE Trans. Instrum. Meas. 40(2), 121 (1991).

${ }^{9}$ G. J. Dick, in Proceedings of the Precise Time and Time Interval (Redondo Beach, CA, 1987), pp. 133-147.

${ }^{10}$ C. Audoin, G. Santarelli, A. Makdissi, and A. Clairon, IEEE Ultrason. Ferroelec. Freq. Control 45(4), 887-894 (1998).

${ }^{11}$ T. M. Fortier, M. S. Kirchner, F. Quinlan, J. Taylor, J. C. Bergquist, T. Rosenband, N. Lemke, A. Ludlow, Y. Jiang, C. W. Oates, and S. A. Diddams, Nat. Photon. 5, 425 (2011).

${ }^{12}$ A. Hati, C. W. Nelson, C. Barnes, D. Lirette, T. Fortier, F. Quinlan, J. A. DeSalvo, A. Ludlow, S. A. Diddams, and D. A. Howe, IEEE Trans. Ultrason. Ferroelectr. Freq. Control 60(9), 1796-1803 (2013).

${ }^{13}$ S. Grop, P. Y. Bourgeois, R. Boudot, Y. Kersalé, E. Rubiola, and V. Giordano, Electron. Lett. 46(6), 420-421 (2010).
${ }^{14}$ D. Chambon, S. Bize, M. Lours, F. Narbonneau, H. Marion, A. Clairon, G. Santarelli, A. Luiten, and M. Tobar, Rev. Sci. Instrum. 76, 094704 (2005)

${ }^{15}$ G. D. Rovera, G. Santarelli, and A. Clairon, IEEE Trans. Ultrason. Ferroelectr. Freq. Control 43(3), 354-358 (1996).

${ }^{16}$ R. Boudot, S. Guérandel, and E. de Clercq, IEEE Trans. Instrum. Meas. 58(10), 3659-3665 (2009).

${ }^{17}$ J. Lautier, M. Lours, and A. Landragin, Rev. Sci. Instrum. 85, 063114 (2014).

${ }^{18}$ See http://www.inrim.it/Mclocks/ for presentation of the MClocks project.

${ }^{19}$ X. Liu, J. M. Merolla, S. Guérandel, E. De Clerq, and R. Boudot, Opt. Express 21(10), 12451-12459 (2013).

${ }^{20}$ See http://www.pascall.co.uk/content/S635144040796327285/pascall20 OCXOoct11.pdf for datasheet of the Pascall OCXO.

${ }^{21}$ E. Rubiola and V. Giordano, Rev. Sci. Instrum. 71(8), 3085 (2000).

${ }^{22}$ E. Rubiola and R. Boudot, IEEE Trans. Ultrason. Ferroelectr. Freq. Control 54(5), 926-937 (2007).

${ }^{23}$ C. W. Nelson, A. Hati, and D. Howe, Rev. Sci. Instrum. 85, 024705 (2014).

${ }^{24}$ See http://literature.cdn.keysight.com/litweb/pdf/5989-6388EN.pdf for basic information on the E5052B signal analyzer.

${ }^{25}$ R. Boudot and E. Rubiola, IEEE Trans. Ultrason. Ferroelectr. Freq. Control 59(12), 2613-2624 (2012).

${ }^{26}$ D. M. Pozar, Microwave Engineering, 3rd ed. (John Wiley and Sons, 2005), Chap. 5 , p. 236

${ }^{27}$ C. W. Nelson, A. Hati, J. F. Garcia-Nava, and D. A. Howe, "Phase noise suppression in frequency comb generators," in Proceedings of the 2010 IEEE International Frequency Control Symposium (IEEE, 2010), pp. 440442.

${ }^{28}$ X. Liu, J. M. Merolla, S. Guérandel, C. Gorecki, E. De Clercq, and R. Boudot, Phys. Rev. A 87, 013416 (2013). 